AperTO - Archivio Istituzionale Open Access dell'Università di Torino

Exploring gold nanoparticles interaction with mucins: A spectroscopic-based study

This is a pre print version of the following article:

Original Citation:

Availability:

This version is available http://hdl.handle.net/2318/1695378

since 2019-03-25T09:55:52Z

Published version:

DOI:10.1016/j.jpharm.2017.11.026

Terms of use:

Open Access

Anyone can freely access the full text of works made available as "Open Access". Works made available under a Creative Commons license can be used according to the terms and conditions of said license. Use of all other works requires consent of the right holder (author or publisher) if not exempted from copyright protection by the applicable law. 


\title{
Exploring gold nanoparticles interaction with mucins: a spectroscopic-based study
}

\author{
Nadia Barbero ${ }^{\mathrm{a}}$, Martina Coletti ${ }^{\mathrm{b}}$, Federico Catalano ${ }^{\mathrm{a}}$, and Sonja Visentin ${ }^{\mathrm{a} *}$ \\ ${ }^{\text {a }}$ Department of Chemistry and NIS Interdepartmental Centre, University of Torino, via Pietro Giuria 7, 10125 Torino, \\ Italy.
}

${ }^{b}$ Molecular Biotechnology and Health Sciences Department, University of Torino, via Quarello 15, 10135 Torino, Italy.

* corresponding author: sonja.visentin@unito.it

\begin{abstract}
The interaction between two mucin types (mucin from porcine stomach - PGM and mucin from bovine submaxillary glands - BSM) and gold nanoparticles (GNPs) of various size (5, 20 and 40 $\mathrm{nm}$ ) and functionalization (with cysteamine or thioglycolic acid) was studied under physiological conditions, in order to investigate the affinity of the nanoparticles to the proteins.

Different methods are employed to monitor the interactions: UV-Visible and fluorescence spectroscopy, fluorescence lifetime, circular dichroism and transmission electron microscopy. These studies have shown the formation of a complex between GNPs and both PGM and BSM.

This aspect could be of great importance for the use of gold nanoparticles for biomedical purposes in those diseases where qualitative and quantitative mucin anomalies play an essential role in mucus composition and rheology.
\end{abstract}

\section{Keywords:}

Gold nanoparticles, mucin, UV-Visible spectroscopy, circular dichroism, fluorescence spectroscopy 


\section{Introduction}

Gold nanoparticles (GNPs) are inert and non-toxic colloidal systems. They are easy to synthesize and have a large surface area (Azam, et al., 2009) which can be exploited to easily functionalize the particles with molecules or biomarkers through thiol linkage (Ghosh et al., 2008). GNPs can be synthesized in different morphologies and size, ranging from 1 to $80 \mathrm{~nm}$, therefore possessing unique physical chemical properties including tunable near-infrared (NIR) localized surface plasmon resonance (SPR) and the ability to bind amine and thiol groups (Jain et al., 2012). Gold nanoparticles have been employed for several decades in many fields (Rashid et al., 2014): imaging (Giordano et al., 2016), bioengineering, biotechnology (Khan et al., 2013) and molecular biology (Jain et al., 2012) whose applications are dependent on the catalytic, electronic, magnetic and optical properties of the particles (Khan et al., 2013). The main application could be their use in cancer diagnosis and therapies (Mesbahi and Carlo, 2010). In fact, it was demonstrated that GNPs contribute to enhance the generation of reactive oxygen species (ROS) such as $\cdot \mathrm{OH}, \mathrm{O}^{2-}$ and ${ }^{1} \mathrm{O}_{2}$, under irradiation of X-rays in the diagnostic range (Ferrero et al., 2017; Strigari et al., 2017). Recently, some authors have described the preparation of gold nanoparticle (GNPs)-based vaccine candidates against the tumor-associated form of the mucin-1 (MUC1) glycoprotein (Cai et al., 2016). Moreover, very recently a group designed fluorescently encoded poly(vinyl alcohol) (PVA)coated gold nanoparticles, functionalized with either negative $\left(-\mathrm{COO}^{-}\right)$or positive $\left(-\mathrm{NH}^{3+}\right)$ surface charges, functionalized with a DC-SIGN antibody on the particle surface, enabling binding to a dendritic cell surface receptor. In this paper a 3D coculture model consisting of epithelial and immune cells (macrophages and dendritic cells) mimicking the human lung epithelial tissue was employed to assess the effects of aerosolized GNPs (Fytianos et al., 2017).

Among issues modulating drug absorption, mucus penetration cannot be ignored. In fact mucus is the first barrier that drugs must overcome to be absorbed and gain access to the circulatory system (Sigurdsson et al., 2013). The major constituents of mucus are water (95-99.5\%) and high molecular weight glycoproteins called mucins. In the mucus, these large oligomeric glycoproteins form networks which are a chemical and physical barrier that not only protects the epithelial but also limits the use of oral administered and inhalatory drugs.

The proof of concept of using mucin to mimic mucus is widely reported in the literature also by Hanes's group, who state that reduced mucin-binding in vitro has been shown to correlate with rapid penetration of nanoparticles through mucus (Xu et al., 2015). Mucin is a large transmembrane 
O-glycosylated protein composed for $75 \%$ of carbohydrate and $25 \%$ of amino acid (Kesari et al., 2015). Mucins are the most abundant macromolecules in mucus, about 20 types have been identified, and are responsible for the biochemical and biophysical properties of mucus (Kesari et al., 2015). An abnormal viscosity and excessive amount of mucus is involved in many respiratory diseases such as cystic fibrosis, asthma and pulmonary diseases (Pontremoli et al., 2015).

In the human body, GNPs have a good distribution and can easily get to the target cells, where they can carry out their action. Once GNPs enter the body, they are exposed to various molecules and can contact and adsorb many proteins such as ubiquitin, serum albumin, tumor necrosis factor (TNF), fibrinogen (Canoa et al., 2015) and cytochrome C. Many studies have investigated these interactions that can induce structural and physiological changes on the biomolecules (Dobrovolskaia et al., 2009); in particular, ubiquitous proteins are able to interact with GNPs and form a protein corona that influences the distribution of nanoparticles in the human body (Wang et al., 2015)

In our previous paper we studied the interaction between CNTs and mucin from porcine stomach Type III (PGM) and it was demonstrated that the superficial functionalization of the material has a great influence on the binding (Barbero et al., 2016). Following these reasons, we studied the interaction between gold nanoparticles and mucin from two commercial mucins, porcine stomach Type III (PGM) and mucin from bovine submaxillary glands (BSM), in order to define their affinity with GNPs. We analysed the interaction with GNPs of different size (5 nm, $20 \mathrm{~nm}$ and $40 \mathrm{~nm})$ and positively and negatively charged GNPs, under physiological conditions. The complex GNPsmucin was characterized by UV-vis and fluorescence spectroscopy, circular dichroism, and transmission electron microscopy (TEM).

\section{Materials and methods}

\subsection{Reagents}

$\mathrm{HAuCl}_{4}$ was purchased from Sigma Aldrich with a purity $\geq 99.9 \%$, the reducing agents trisodium citrate and $\mathrm{NaBH}_{4}$ produced by Sigma Aldrich had a purity $\geq 99 \%$ and $\geq 98 \%$ respectively.

Cysteamine and thioglycolic acid were purchased from Sigma Aldrich with a purity $\geq 98 \%$ and were used to functionalize gold nanoparticles. The solutions employed for the synthesis were prepared by dissolving the reagents in Millipore water. 
Mucin from porcine stomach (PGM) (Type III, bound sialic acid 0.5-1.5\%, partially purified powder) was purchased from Sigma Aldrich. PGM stock solution (1 mg/mL) was prepared by dissolving the protein in PBS (phosphate buffer solution, $2 \mathrm{mM}, \mathrm{pH}=7.4$ ).

Mucin from bovine submaxillary glands (BSM, Type I-S) was purchased from Sigma Aldrich. BSM stock solution $(1 \mathrm{mg} / \mathrm{mL})$ was prepared by dissolving the protein in PBS (phosphate buffer solution, $2 \mathrm{mM}, \mathrm{pH}=7.4)$.

\subsection{Synthesis and functionalization of GNPs}

Gold colloidal nanoparticles, with three different sizes 5, 20, $40 \mathrm{~nm}$, were synthesized with the Turkevich's method by varying the ratio between trisodium citrate in order to obtain various diameters (Daniel and Astruc, 2004).

To synthesize GNPs with a size of about $5 \mathrm{~nm}, 10 \mathrm{~mL}$ Millipore water solution of the gold salt $(0.5$ $\mathrm{mM}$ ) were added to $10 \mathrm{~mL}$ Millipore water solution of trisodium citrate $(0.6 \mathrm{mM})$; then $0.6 \mathrm{~mL}$ of a water solution of $\mathrm{NaBH}_{4}(0.1 \mathrm{M})$ was added to the solution.

$15.4 \mathrm{~mL} \mathrm{NaOH} 20 \mathrm{mM}$ and $19.6 \mathrm{~mL}$ Millipore water were added to $5 \mathrm{~mL}$ of a $25 \mathrm{mM} \mathrm{HAuCl}_{4}$ solution in Millipore water. The solution was heated for 30 minutes at $110^{\circ} \mathrm{C} \cdot 1.2 \mathrm{~mL}$ Millipore solution of trisodium citrate $170 \mathrm{mM}$ are then added and the sample was again left for 15 minutes at $110^{\circ} \mathrm{C}$. GNPs with a diameter of $20 \mathrm{~nm}$ were obtained.

To obtain GNPs with $40 \mathrm{~nm}$ of diameter, the citrate to gold salt mole ratio was 1.36 and $1.5 \mathrm{~mL}$ of $1 \%$ trisodium citrate was added to $250 \mathrm{~mL} \mathrm{HAuCl}_{4} 0,25 \mathrm{mM}$ in Millipore water solution.

Gold nanoparticles with a diameter of $20 \mathrm{~nm}$ were functionalized with cysteamine (Cys) and thioglycolic acid (Tga) to shape GNPs with a positive and a negative charge, respectively. To 10 $\mathrm{mL}$ GNPs $1 \mathrm{mM}$ were added $500 \mu \mathrm{L}$ Millipore water solution of cysteamine $0.26 \mathrm{mM}$. To $10 \mathrm{~mL}$ GNPs $1 \mathrm{mM}$ were added $15 \mu \mathrm{L}$ Millipore water solution of thioglycolic acid $0.26 \mathrm{mM}$.

The GNPs dimension was checked by UV-vis spectroscopy and TEM (see figure S1 SI).

\subsection{GNP-protein complex formation and purification}

1.5-4.5 $\mathrm{ml}$ of GNPs $(1 \mathrm{mM})$ were added to a $0.5 \mathrm{~mL}$ solution of mucin protein $(1 \mathrm{mg} / \mathrm{mL})$ in $2 \mathrm{mM}$ sodium phosphate buffer, $\mathrm{pH} 7.2$ and the mixture was stirred for $1 \mathrm{~h}$ at room temperature. The reaction mixture was then loaded on a Sephadex G-25 gel-permeation column. The protein complexes were eluted with a sodium phosphate buffer and the elution was monitored by UV/Vis spectroscopy. 


\subsection{Instrumentation}

\subsubsection{UV-Visible and fluorescence spectroscopy}

UV-Visible measurements were recorded using a UH5300 Hitachi spectrophotometer. Increasing concentrations of GNPS (ranging $10 \mu \mathrm{M}-200 \mu \mathrm{M}, 10 \mu \mathrm{M}-400 \mu \mathrm{M}$ and $10 \mu \mathrm{M}-450 \mu \mathrm{M}$ for GNPs, GNPs-Cys and GNPs-Tga respectively) were added to a constant concentration of PGM $(0.05 \mathrm{mg} / \mathrm{mL})$ or BSM $(0.15 \mathrm{mg} / \mathrm{mL})$.

Fluorescence spectra were recorded with a Horiba Jobin Yvon Fluorolog2. Emission spectra were recorded in the range of $280-500 \mathrm{~nm}$ after excitation at $258 \mathrm{~nm}$. The excitation and emission slits were $6 \mathrm{~nm}$ and $10 \mathrm{~nm}$ respectively. A constant concentration of mucin $(0.1 \mathrm{mg} / \mathrm{mL}$ both for PGM and BSM) was analyzed by successive increasing additions of GNPs (ranging $10 \mu \mathrm{M}-350 \mu \mathrm{M}, 10$ $\mu \mathrm{M}-300 \mu \mathrm{M}$ and $10 \mu \mathrm{M}-350 \mu \mathrm{M}$ for GNPs, GNPs-Cys and GNPs-Tga respectively).

Fluorescence lifetimes were obtained using a NanoLED source $(297 \mathrm{~nm})$ and a photon counting detector (TBX04). The goodness of the fit was assessed by the chi-squared value of less than 1.2 and a residual trace that was symmetric about the zero axes. The final result is an average of three independent measurements.

\subsubsection{Circular Dichroism}

UV-Circular Dichroism (CD) spectroscopy was used to evaluate changes in the secondary structure of PGM and BSM mucin after irreversible adsorption on bare and functionalized GNPs. Watery protein solutions $(1 \mathrm{mg} / \mathrm{mL})$ and suspensions of purified mucin-GNPs complexes were scanned in the far-UV spectral range (four accumulations) over the wavelength region 180-260 nm with a scanning speed of $50 \mathrm{~nm} \mathrm{m^{-1 }}$ using a Jasco J-815 spectropolarimeter equipped with a Xe arc lamp. Spectra were recorded in a quartz cuvette (optical path length $0.1 \mathrm{~cm}$ ). Baseline was subtracted for each measurement. Spectra of adsorbed mucin were normalized with respect the one of native protein according to the absorbance in the wavelength region where electronic transitions occur. After normalization, spectra were compared at the same nominal concentration $(1 \mathrm{mg} / \mathrm{mL})$.

Secondary structure was estimated from the ellipticity $[\vartheta, \mathrm{mdeg}]$ with the CDNN CD spectra deconvolution software [Version 2.1, Copyright (C) 1997 Gerald Böhm] and for each sample, a maximum standard deviation of 5\% in the $185-260 \mathrm{~nm}$ spectral range was considered. The declared 
root mean square errors (r.m.s. x 100) in the wavelength range considered for this work (180-260 $\mathrm{nm})$ is 2.0987 and the average error (\%) of predicted secondary structures of the network is 3.98 .

\subsubsection{Transmission electron microscopy}

High-resolution transmission electron micrographs were obtained with a JEOL 3010-UHR instrument operating at $300 \mathrm{kV}$, equipped with a $2 \mathrm{k} \times 2 \mathrm{k}$ pixels Gatan US1000 CCD digital camera. Samples of purified mucin-GNPs complex were dried on a carbon-coated copper grid. The nominal magnification used to record the complex mucin-GNPS were x500000 and x800000.

\section{Results and discussion}

\subsection{UV-Visible spectroscopy}

UV-Visible measurements were performed in order to investigate the interaction between GNPs and mucin. The spectra were obtained keeping constant the concentration of PGM $(0.05 \mathrm{mg} / \mathrm{mL})$ in presence or absence of GNPs with a diameter of $5 \mathrm{~nm}, 20 \mathrm{~nm}$ and $40 \mathrm{~nm}$. Spectra of samples with an increasing concentration of GNPs showed an increased adsorption intensity compared to mucin alone (black line for both PGM and BSM), whose peak is at $258 \mathrm{~nm}$ (see SI for spectra). This behaviour confirmed the formation of a complex between the nanostructures and the protein. The same method was employed to analyse the formation of a complex between PGM or BSM and GNPs-cysteamine or GNPs-thioglycolic acid. In both cases, the intensity absorption of the protein alone, at around $260 \mathrm{~nm}$, increased with an increasing concentration of GNPs-cysteamine or GNPsthioglycolic acid. Moreover, in the case of thioglycolic acid there was also a slight ipsochromic shift, further confirming the formation of a complex.

\subsection{Fluorescence spectroscopy}

Both PGM and BSM mucins showed an intensive fluorescence at about $360 \mathrm{~nm}$, upon excitation at $258 \mathrm{~nm}$. Fluorescence spectra are reported in Fig 1a and show a gradually decrease of the PGM emission intensity and a slight bathochromic shift, caused by successive additions of increased concentrations of GNPs with a diameter of $20 \mathrm{~nm}$ (see SI for fluorescence spectra of further analyses with GNPs of $5 \mathrm{~nm}$ and $40 \mathrm{~nm}$ and functionalized GNPs with cysteamine and thioglycolic acid). 

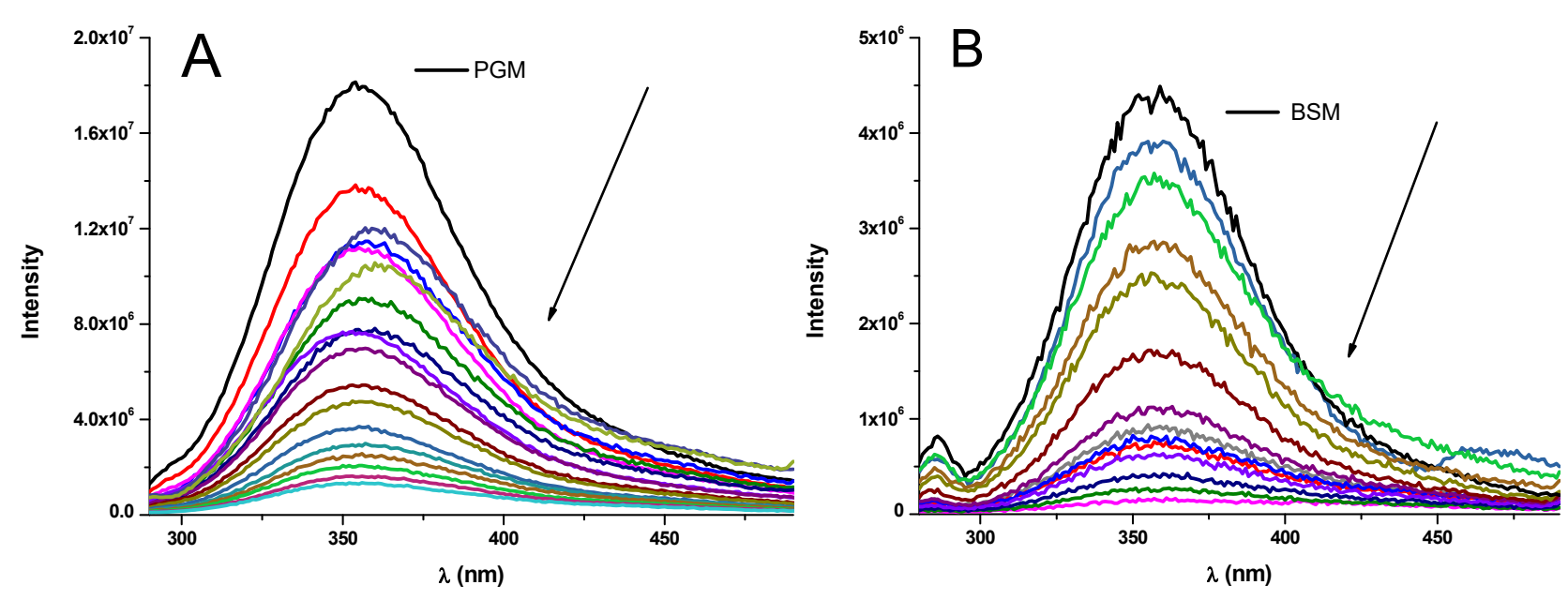

Figure 1 : A) quenching fluorescence of PGM $(0.1 \mathrm{mg} / \mathrm{mL})$ in presence of increasing concentrations of GNPs of $20 \mathrm{~nm}(10,15,20,30,35,40,50,60,70,80,90,100,110,120,130$, $140,150 \mu \mathrm{M})$; B) quenching fluorescence of BSM $(0.1 \mathrm{mg} / \mathrm{mL})$ in presence of increasing concentrations of GNPs of $20 \mathrm{~nm}(10,30,60,90,100,120,150,170,200,250,270,300,350 \mu \mathrm{M})$.

A decrease in the fluorescence signal was also obtained for BSM protein (Fig. 1b) after the addition of increasing concentrations of GNPs with a diameter of $20 \mathrm{~nm}$ (see SI for fluorescence spectra of further analyses with GNPs of $5 \mathrm{~nm}$ and $40 \mathrm{~nm}$ and functionalized GNPs with cysteamine and thioglycolic acid).

The quenching of the emission intensity of mucin was caused by the interaction between the protein and the nanoparticles confirming the UV-Vis data. Once the complex formation was assessed, binding studies were performed and the obtained steady-state maximum fluorescence intensity was recorded.

\subsubsection{Data analysis}

The obtained spectroscopic data were analysed by different methods allowing to obtain the association and dissociation constants of the interactions, $K_{a}$ and $K_{d}$ respectively. The fluorescence quenching of mucin was analysed by Stern-Volmer equation (Pontremoli et al., 2017), Eq. 1:

$$
\frac{F_{0}}{F}=1+K s v[Q]
$$

where $\mathrm{F}_{0}$ is the fluorescence intensity of mucin alone and $\mathrm{F}$ is the fluorescence of mucin together with an increased concentration of quencher [Q], i.e. the gold nanoparticles. Ksv describes the 
collisional quenching of fluorescence. Quenching data are presented as plot of $\mathrm{F}_{0} / \mathrm{F}$ versus [Q], yielding an intercept of one on the $\mathrm{Y}$-axis and a slope equal to $\mathrm{K}_{\text {sv }}$. Fluorescence data were also analysed by Lineweaver-Burk equation (Pontremoli et al., 2017), Eq. 2:

$$
\frac{1}{F_{0}-F}=\frac{1}{F_{0}}+\frac{K_{d}}{F_{0}[Q]}
$$

where $F_{0}$ is the steady-state fluorescence intensity of mucin alone and $F$ is the steady-state fluorescence intensity of mucin in the presence of increased concentration of quencher, [Q] is the gold nanoparticles concentration and $K_{d}$ is the equilibrium dissociation constant. In the graphs, reciprocals of $F_{0}-F$ are plotted versus reciprocals of [Q]. The slope of the line is $K_{d} / F_{0}$ ratio while the intercept is the reverse of $F_{0}$. The association constant $K_{a}$ is calculated from $K_{d}$. The last equation used to analyse the experimental data is non linear least-squares fit procedures (Pontremoli et al., 2017), Eq. 3:

$$
y=\frac{B_{\max }[Q]}{K_{d}+[Q]}
$$

in which $\mathrm{y}$ is the binding derived by measuring fluorescence intensity, [Q] is gold nanoparticles concentration, $\mathrm{B}_{\max }$ is the maximum quantity of GNPS-mucin complex formed at saturation and $\mathrm{K}_{\mathrm{d}}$ is the equilibrium dissociation constant. The percentage of bound protein is plotted against [Q], GNPS concentration. The association constant $\mathrm{K}_{\mathrm{a}}$ is calculated from $\mathrm{K}_{\mathrm{d}}$. As an example, Fig. 2 represents the data analysis of a sample with GNPs and mucin from porcine stomach, Type III (the data analysis of all the other samples are reported in the SI).

The corresponding values of the Stern-Volmer constants $\left(\mathrm{M}^{-1}\right)$ obtained from the data analysis and the values of association $\left(\mathrm{K}_{\mathrm{a}}\right)$ and dissociation $\left(\mathrm{K}_{\mathrm{d}}\right)$ constants are reported in Table 1 and 2.
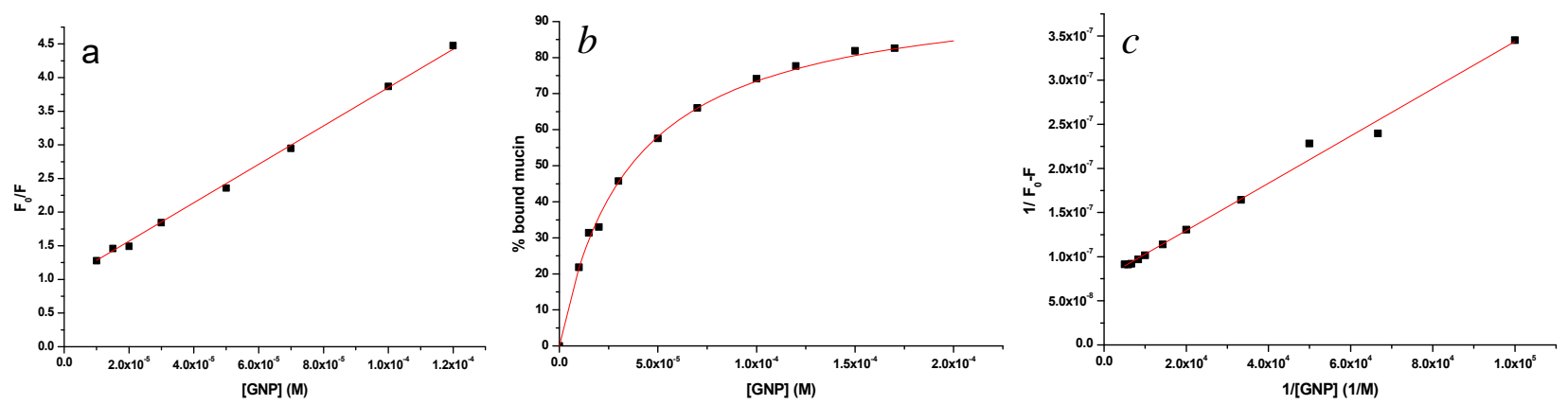

Figure 2: data analysis of sample PGM-GNPs (40 nm) by Stern-Volmer equation (a), by non linear fit (b), by Lineweaver-Burk equation (c). 
Table 1: values of quenching costants and equilibrium association and dissociation constants of PGM and GNPs: GNPs of $5 \mathrm{~nm}$, GNPs of $20 \mathrm{~nm}$, GNPs of $40 \mathrm{~nm}$, GNPs-Cys and GNPs-Tga.

\begin{tabular}{|c|c|c|c|c|c|c|}
\hline PGM & & $\begin{array}{l}\text { GNPs } \\
5 \mathrm{~nm}\end{array}$ & $\begin{array}{l}\text { GNPs } \\
20 \mathrm{~nm}\end{array}$ & $\begin{array}{l}\text { GNPs } \\
40 \mathrm{~nm}\end{array}$ & GNPs-Cys & GNPs-Tga \\
\hline Stern-Volmer equation & $K_{s v}\left(M^{-1}\right)$ & $6.48 \times 10^{4}$ & $4.34 \times 10^{4}$ & $2.80 \times 10^{4}$ & $6.74 \times 10^{3}$ & $8.57 \times 10^{3}$ \\
\hline Non linear fit equation & $\begin{array}{l}K_{d}(M) \\
K_{a}\left(M^{-1}\right)\end{array}$ & $\begin{array}{l}5.12 \times 10^{-5} \\
1.95 \times 10^{4}\end{array}$ & $\begin{array}{l}3.83 \times 10^{-5} \\
2.60 \times 10^{4}\end{array}$ & $\begin{array}{l}3.95 \times 10^{-5} \\
2.79 \times 10^{4}\end{array}$ & $\begin{array}{l}1.33 \times 10^{-4} \\
7.52 \times 10^{3}\end{array}$ & $\begin{array}{l}1.19 \times 10^{-4} \\
8.40 \times 10^{3}\end{array}$ \\
\hline $\begin{array}{l}\text { Lineweaver-Burk } \\
\text { equation }\end{array}$ & $\begin{array}{l}K_{d}(M) \\
K_{a}\left(M^{-1}\right)\end{array}$ & $\begin{array}{l}4.92 \times 10^{-5} \\
2.03 \times 10^{4}\end{array}$ & $\begin{array}{l}3.71 \times 10^{-5} \\
2.69 \times 10^{4}\end{array}$ & $\begin{array}{l}3.54 \times 10^{-5} \\
2.81 \times 10^{4}\end{array}$ & $\begin{array}{l}1.24 \times 10^{-4} \\
8.00 \times 10^{3}\end{array}$ & $\begin{array}{l}9.50 \times 10^{-5} \\
1.08 \times 10^{4}\end{array}$ \\
\hline
\end{tabular}

Table 2: values of quenching costants and equilibrium association and dissociation constants of BSM with GNPs of $20 \mathrm{~nm}$, GNPs-Cys, GNPs-Tga.

\begin{tabular}{|c|c|c|c|c|}
\hline BSM & & GNPs 20 nm & GNPs-Cys & GNPs-Tga \\
\hline Stern-Volmer equation & $K_{s v}\left(M^{-1}\right)$ & $5.00 \times 10^{4}$ & $1.88 \times 10^{3}$ & $3.04 \times 10^{4}$ \\
\hline Non linear fit equation & $\begin{array}{l}K_{d}(M) \\
K_{a}\left(M^{-1}\right)\end{array}$ & $\begin{array}{l}6.70 \times 10^{-5} \\
1.49 \times 10^{4}\end{array}$ & $\begin{array}{l}6.27 \times 10^{-5} \\
1.59 \times 10^{4}\end{array}$ & $\begin{array}{l}4.48 \times 10^{-5} \\
2.23 \times 10^{4}\end{array}$ \\
\hline Lineweaver-Burk equation & $\begin{array}{l}K_{d}(M) \\
K_{a}\left(M^{-1}\right)\end{array}$ & $\begin{array}{l}5.02 \times 10^{-5} \\
1.99 \times 10^{4}\end{array}$ & $\begin{array}{l}5.42 \times 10^{-5} \\
1.84 \times 10^{4}\end{array}$ & $\begin{array}{l}4.17 \times 10^{-5} \\
2.11 \times 10^{4}\end{array}$ \\
\hline
\end{tabular}

$\mathrm{K}_{\mathrm{a}}$ and $\mathrm{K}_{\mathrm{d}}$ are used to find out the affinity between gold nanoparticles and the two mucin types. Similar equilibrium values have been obtained employing different data processing methods: SternVolmer equation, non linear fit equation and Lineweaver-Burk equation.

The analysis of equilibrium dissociation constants between GNPs of various size $(5,20,40 \mathrm{~nm})$ and PG mucin showed values with the same order of magnitude. This means that the size of the gold nanoparticle does not influence the interaction with the protein significantly. Instead the binding constants change if the interaction between functionalized GNPs and PG mucin occurs. As regards the nanoparticles positively charged with cysteamine and PGM, $\mathrm{K}_{\mathrm{d}}$ is greater compared to the nonfunctionalized ones and so the affinity is lower. The negatively charged GNPs with thioglycolic acid show a dissociation value with the PGM similar to GNPs-Cys, the affinity with the protein is slightly higher than the positive ones. The analysis of dissociation values between GNPs and BS Mucin revealed similar constants for both non- functionalized nanoparticles of $20 \mathrm{~nm}$ that for those 
functionalized ones. Moreover, $\mathrm{K}_{\mathrm{d}}$ has the same order of magnitude compared to the values found by the interaction GNPs-PGM. In conclusion, regarding the analysis with BS mucin, the functionalization of the nanoparticles and the charge does not affect significantly on the interaction with the protein.

\subsubsection{Lifetime}

The fluorescence lifetimes of mucins in the presence of nanoparticles were measured using the tryptophan aminoacid residues in the protein structure which have an emission maximum of 360 $\mathrm{nm}$. Lifetimes were analyzed for mucin (PGM or BSM) alone and then for samples consisted of the same concentration of mucin and the concentration of gold nanoparticles at saturation. The fluorescence decays of both mucins were fitted by a three exponential equation; with $\tau 1=1.3 \mathrm{~ns}$, $\tau 2=3.3 \mathrm{~ns}$ and $\tau 3=7.0 \mathrm{~ns}$ for PGM. The first component is responsible for around $64 \%$ of the total intensity decay (for BSM values see SI). When mucins are excited in the presence of GNPs, $\tau 1$ is always reduced reaching for example a value of $0.5 \mathrm{~ns}$ in the case of PGM interacting with GNPcysteamine. The same behaviour is observed for all the studied interactions; for a complete analysis see table S1 in the SI. Lifetime measurements are the most definitive method to distinguish static and dynamic quenching. Analyzing the lifetimes in the absence and in the presence of gold nanoparticles, some changes in the decay times can be appreciated for both PG and BS mucin confirming a collisional quenching. This quenching behavior is also evident in UV-vis spectroscopy where no changes (shift and shape) are observed (see SI). This could be explained by the fact that collisional quenching only affects the excited states of the fluorophores (Lakowicz, 2006).

\subsection{Transmission Electron Microscopy}

In order to obtain direct evidence on protein corona formation on the GNPs surface, morphological analysis was performed using TEM. Fig. 3 reports the images obtained from TEM analysis revealing a thin shell of mucin formed over the GNPs. 

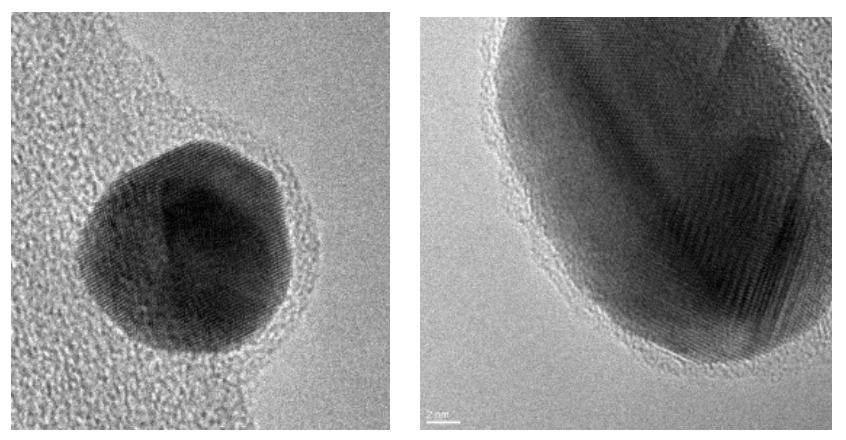

Figure 3: images of complex GNPs-PGM seen under the transmission electron microscope with a magnification of x500000 and x800000.

\subsection{Circular Dichroism}

Spectra of PG and BS mucin, both in native and adsorbed forms, were acquired and reported in Figure 4. Despite some differences due to the different MW of the two proteins, they both present the typical CD spectrum of proteins mainly composed by unordered secondary structures, characterized by a negative band at 205-200 nm due to $\pi-\pi^{*}$ electronic transition (Yang et al., 2005). If compared with their reference (protein in native form), the spectra of mucin adsorbed on bare and functionalized GNPs did not reveal significant changes in the spectra profiles. Slight changes can be appreciated in the relative intensities of BSM adsorbed on GNPs if compared to BSM in native form (Fig. 4). In order to quantitatively estimate such differences, CD spectra deconvolution were performed (the deconvolutions obtained are shown in SI). Despite the change in the minima at 207 and $200 \mathrm{~nm}$ for PGM and BSM, respectively, they both are composed by similar percentage of secondary structural motifs, with a predominance of random coils. Passing to the adsorbed proteins, a general increase of $\beta$-sheet like motifs is observed, indicative of the formation of a layer of proteins in interaction on the surface of GNPs. This is in agreement with the TEM observation, where a corona layer can be observed independently by the surface functionalization of GNPs. 

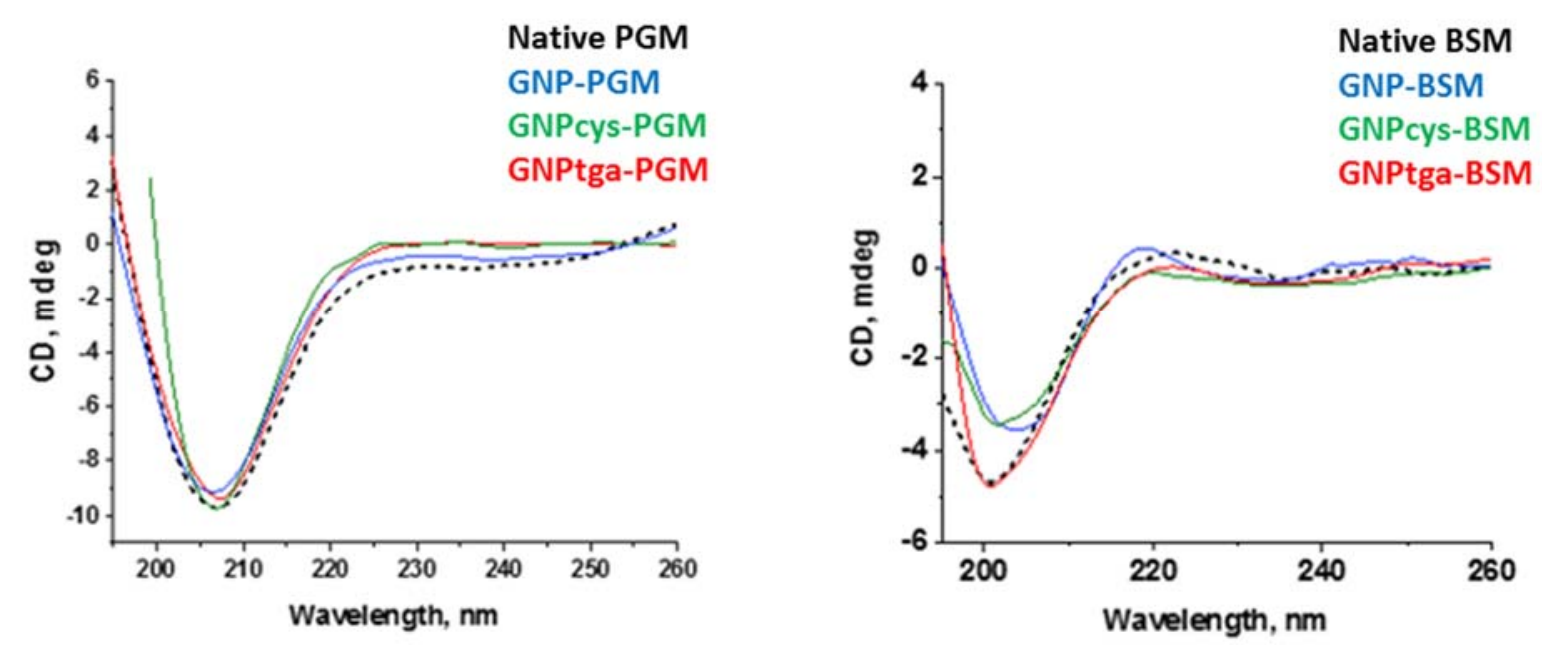

Figure 4: spectra of both mucins and their complex with GNPs by circular dichroism

\section{Conclusions}

In this paper, the interaction between two mucin types (mucin from porcine stomach - PGM and mucin from bovine submaxillary glands - BSM) and gold nanoparticles of different size (5, 20 and $40 \mathrm{~nm}$ ) and with different functionalization was investigated by using various methods. The formation of a complex between both kind of proteins and nanomaterials was assessed by UVVisible spectroscopy, where the increase of mucin absorption with the addition of GNPs underlines the formation of the complex. This was further proved by fluorescence spectroscopy, in which the quenching of mucin intensity is related to the presence of a formed GNPs-protein complex. These findings clearly demonstrate that the microenvironment of mucin was changed during the binding interaction with all the NPs used.

Circular dichroism was used to investigate possible changes in the secondary structure of the protein after interaction with gold nanoparticles: spectra showed no changes in the structure, despite the use of GNPs with different sizes.

To support and define the formation of a complex, samples were analyzed with TEM and the obtained images have shown a mucin layer around the nanoparticles.

The present results may be of the utmost importance for the use of gold nanoparticles for biomedical purposes, in those diseases characterized by the presence of mucus. Next step will be to study the behavior of nanoparticles in contact with mucus, whose composition approaches and mimics the pathological one. 


\section{Acknowledgements}

Authors acknowledge the financial support from the University of Torino (Ricerca Locale ex-60\%, Bando 2016).

\section{References and notes}

Azam, A., Ahmed, F., Arshi, N., Chaman, M., \& Naqvi, A. H. 2009. One step synthesis and characterization of gold nanoparticles and their antibacterial activities against E . coli (ATCC 25922 strain), 2-5. International Journal of Theoretical \& Applied Sciences, 1(2): 1-4

Barbero, N., Marenchino, M., Luca, S. O., Jasminka, B., Viscardi, G., \& Visentin, S. 2016. Nanomaterial - protein interactions : the case of pristine and functionalized carbon nanotubes and porcine gastric mucin. J. Nanoparticles Research 18: 179-185.

Wang, P., Wang, X., Wang, L., Hou, X., Liu, W, \& Chen, C..2015. Interaction of gold nanoparticles with proteins and cells. Science and Technlogy of Advanced Materials, 16, 034610.

Cai, H., Degliangeli, F., Palitzsch, B., Gerlitzki, B., Kunz, H., Schmitt, E., Fiammengo, R., Westerlind, U. 2016. Glycopeptide-functionalized gold nanoparticles for antibody induction against the tumor associated mucin-1 glycoprotein. Bioorganic \& Medicinal Chemistry, 24, $1132-1135$.

Canoa, P., Simón-Vázquez, R., \& Popplewell, J. 2015. Biosensors and Bioelectronics A quantitative binding study of fi brinogen and human serum albumin to metal oxide nanoparticles by surface plasmon resonance. Biosensors and Bioelectronic, 74, 376-383.

Daniel, M., \& Astruc, D. 2004. Gold Nanoparticles : Assembly, Supramolecular Chemistry, Quantum-Size-Related Properties and Applications toward Biology, Catalysis and Nanotechnology.Chemical Review 104(1):293-346

Dobrovolskaia, M. A., Patri, A. K., Zheng, J., Clogston, J. D., Ayub, N., Aggarwal, P., Neun, B.W., Hall, J.B., Mcneil, S. E. 2009. Interaction of colloidal gold nanoparticles with human blood: effects on particle size and analysis of plasma protein binding profiles. Nanomedicine 5, 106117.

Ferrero, V., Visonà, G., Dalmasso, F., Gobbato, A.,Cerello, P., Strigari, L., Visentin, S., Attili, A., 2017. Targeted dose enhancement in radiotherapy for breast cancer using gold nanoparticles, part 1: A radiobiological model study. Med Phys 44, 1983-1992. 
Fytianos, K., Chortarea, S., Rodriguez-Lorenzo, R., Blank, F., von Garnier, C., Petri-Fink, A., \& Rothen-Rutishauser, B. 2017. Aerosol Delivery of Functionalized Gold Nanoparticles Target and Activate Dendritic Cells in a 3D Lung Cellular Model. ACS Nano, 11, 375-383.

Ghosh, P., Han, G., De, M., Kim, C. K., \& Rotello, V. M. 2008. Gold nanoparticles in delivery applications. Advanced Drug Delivery Reviews, 60, 1307-1315.

Giordano, S., Morosi, L., Veglianese, P., Licandro, S. A., Frapolli, R., Zucchetti, M., Cappelletti, G., Falciola, L., Pifferi, V., Visentin, S., D'Incalci, M., Davoli, E., 2016. 3D Mass Spectrometry Imaging Reveals a Very Heterogeneous Drug Distribution in Tumors. Sci Rep, 6,37027 .

Jain, S., Hirst, D. G., \& O’Sullivan, J. M. 2012. Gold nanoparticles as novel agents for cancer therapy, Br .J. Radiol 85, 101-113.

Kesari, M. V., Gaopande, V. L., Joshi, A. R., Babanaqare, S.V.,Goqate, B.P., Khadilkar, A.V., 2015. Immunohistochemical study of MUC1 , MUC2 and MUC5AC in colorectal carcinoma and review of literature. Indian. J. Gastroenterology 34, 63-67.

Khan, Z., Singh, T., Hussain, J. I., \& Hashmi, A. A. 2013. Au ( III )- CTAB reduction by ascorbic acid : Preparation and characterization of gold nanoparticles. Colloids and Surfaces B: Biointerfaces, 104, 11-17.

Lakowicz JR. Principles of fluorescence spectroscopy. Springer- Verlag: New York, 2006.

Mesbahi, A. 2010. A review on gold nanoparticles radiosensitization effect in radiation therapy of cancer. Reports of Practical Oncology and Radiotherapy, 15, 176-180.

Pontremoli, C., Barbero, N., Viscardi, G., \& Visentin, S. 2015. Mucin - drugs interaction : The case of theophylline, prednisolone and cephalexin. Bioorganic \& Medicinal Chemistry, 23, 65816586.

Pontremoli, C., Barbero N.,Viscardi G., \& Visentin, S. 2017. Insight into the interaction of Inhaled Corticosteroids (ICS) with human serum albumin: A spectroscopic-based study Journal of Pharmaceutical Analysis, in press https://doi.org/10.1016/j.jpha.2017.07.003

Khan, A.K., Rashid, R., Murtaza, G., \& Zahra, A. 2014. Gold Nanoparticles: Synthesis and Applications in Drug Delivery, Tropical Journal of Pharmaceutical Research 13, 1169-1177.

Sigurdsson, H. H., Kirch, J., \& Lehr, C. 2013. Mucus as a barrier to lipophilic drugs. International 
Journal of Pharmaceutics, 453, 56-64.

Strigari, L., Ferrero, V., Visonà, G., Dalmasso, F., Gobbato, A., Cerello, P., Visentin, S., Attili, A., 2017. Targeted dose enhancement in radiotherapy for breast cancer using gold nanoparticles, part 2: A treatment planning study. Med Phys 44, 1993-2001.

Xu, Q., Ensign, L. M., Boylan, N. J., Schön, A., Gong, X., Yang, J.C., Lamb, N.W., Cai, S., Yu, T., Freire, E., Hanes, J. 2015. Impact of Surface Polyethylene Glycol (PEG) Density on Biodegradable Nanoparticle Transport in Mucus ex Vivo and Distribution in Vivo, ACS Nano 9, 9217-9227.

Yang, L. M., Wang, Y. J., Luo, G. S., \& Dai, Y. Y. 2005. Simultaneous removal of copolymer template from SBA-15 in the crystallization process, Microporous and Mesoporous Materials 81, 107-114. 